\title{
Analog Line Driver with Adaptive Impedance Matching
}

\author{
Bram Nauta, Member, IEEE, and Marcel B. Dijkstra
}

\begin{abstract}
A new principle for an adaptive line driver is presented. This type of line driver can adapt its output impedance automatically to the applied load. This results in automatically corrected output impedance for different cables with terminations. Also, the line-driver output impedance becomes insensitive to process variations. As an example, a line driver for analog video signals has been designed. The circuit operates from a 2.4$V$ supply in a $0.35-\mu \mathrm{m}$ CMOS technology. The realized circuit adapts between 38 and $85 \Omega$ loads, has total harmonic distortion of $<-50 \mathrm{~dB}$ at $1.2 \mathrm{~V}_{\mathrm{pp}}$ for $0-10 \mathrm{MHz}, 0.09-\mathrm{mm}^{2}$ area, and 9-mW static power consumption.
\end{abstract}

Index Terms-Adaptive circuits, analog circuits, line driver.

\section{INTRODUCTION}

$\mathbf{T}$ RANSMISSION lines are widely used for transportation of electrical signals. To minimize reflections, the source and load impedances of the transmission line have to be equal to the characteristic impedance of the line. For example, analog video applications typically use 75 and $50 \Omega$ as a standard for instrumentation.

Fig. 1 shows two basic ways to drive a transmission line. In the configuration of Fig. 1(a), the line is driven by a voltage source with series resistance $R_{s}$ [1]. The disadvantage of this configuration is that half the voltage $V_{\text {in }}$ is lost across $R_{s}$. If an output voltage $V_{\text {out }}$ of $1.5 \mathrm{~V}$ is desired, $V_{\text {in }}$ must be $3 \mathrm{~V}$. This becomes a problem if the supply voltage is lower than $3 \mathrm{~V}$, which is the case for modern deep submicrometer CMOS processes. For low supply voltages, the configuration of Fig. 1(b) [2] is more attractive. Here, the line driver is replaced by its Norton equivalent, and no signal voltage is lost. However, half the signal current is lost through $R_{s}$. A $1.5-\mathrm{V}$ signal across an $R_{\mathrm{s}}$ of $75 \Omega$ results in a current loss of $20 \mathrm{~mA}$, which is unattractive. If the resistor is integrated, both configurations have another disadvantage, which is sensitivity to process variations. Typical numbers for the spread of a polyresistor are $\pm 30 \%$, resulting in $\pm 30 \%$ spread in line-driver output impedance; and $\pm 15 \%$ spread in $V_{\text {out }}$.

This paper describes a line driver without voltage or current loss in $R_{s}$. Furthermore, the line driver adapts its output impedance automatically to a value equal to the applied

Manuscript received April 28, 1998; revised July 21, 1998.

B. Nauta was with Philips Research Laboratories, Eindhoven, The Netherlands. He is now with the University of Twente, Enschede 7500 AE The Netherlands.

M. B. Dijkstra was with Philips Research Laboratories, Eindhoven, The Netherlands. He is now with Philips Optical Recording Laboratories, Eindhoven, The Netherlands.

Publisher Item Identifier S 0018-9200(98)08863-5.
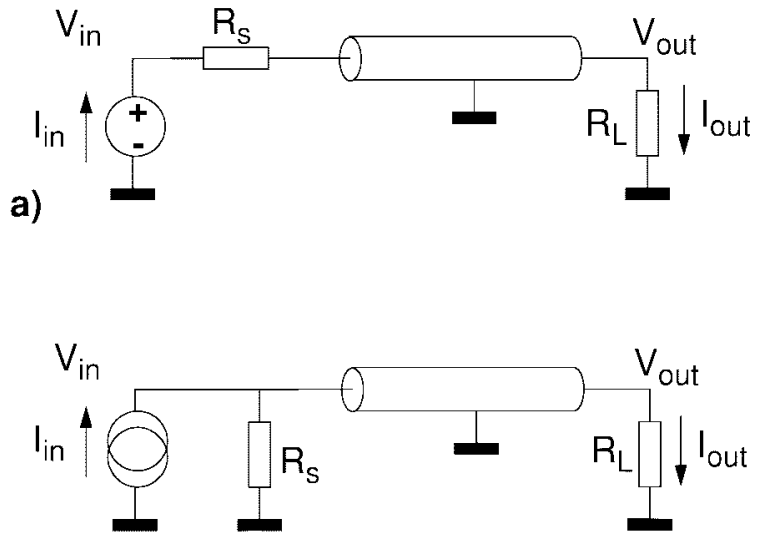

b)

Fig. 1. Two classical ways to drive a transmission line: (a) Voltage source with series resistor $R_{s}$, resulting in voltage loss across $R_{s}$. (b) Current source with parallel resistor $R_{s}$, resulting in current loss in $R_{s}$.

load. The advantages are impedance insensitivity to process variations and automatic adaptation of its output impedance if another load is applied. Consequently, if another (well terminated) cable with another characteristic impedance is connected, the line driver automatically tunes to the correct output impedance. For transporting digital signals over printed circuit boards, adaptive line drivers do exist [3], [4]. However, these need external components, a complex control scheme, and/or a separate return path from the receiving side. Moreover, these drivers are not suited for analog signals. The line driver presented in this paper is intended for analog signals.

In Section II, the basic idea of the line driver is explained. In Section III, this basic idea is applied in an analog baseband video line driver. In Section IV, the experimental results are given and conclusions are drawn.

\section{BASIC IDEA}

Fig. 2 illustrates the new line-driver architecture. For the sake of understanding, the line driver is explained in three steps. The first step in Fig. 2(a) shows a linear transconductor $g m_{1}$ with transconductance $g m$ driving the line, which is modeled by a resistor $R_{L}$. The output current $g m \cdot V_{\text {in }}$ is fed into $R_{L}$, resulting in an output voltage $V_{\text {out }}=g m \cdot V_{\text {in }} \cdot R_{L}$. Suppose the transconductance $g m$ is electronically tunable via a "gm-control voltage" $V_{\text {control }}$ and that by some means, $g m$ is tuned to be equal to $1 / R_{L}$. In that case, the voltage gain 


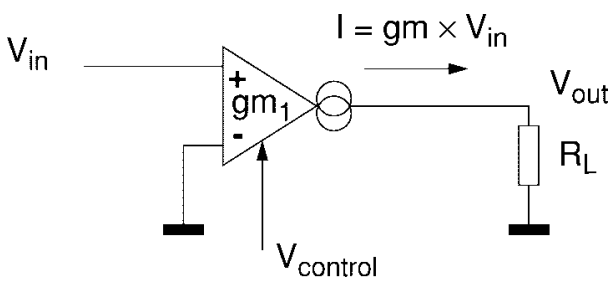

(a)

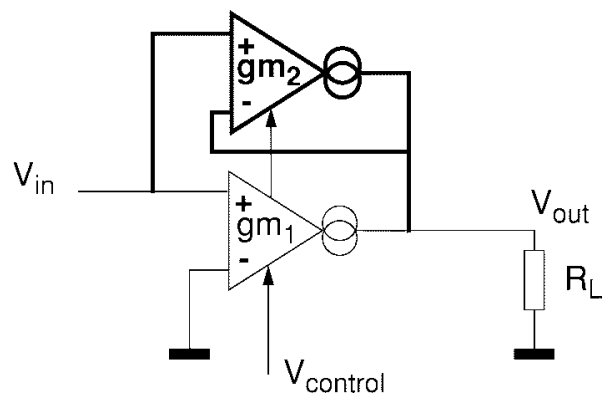

(b)

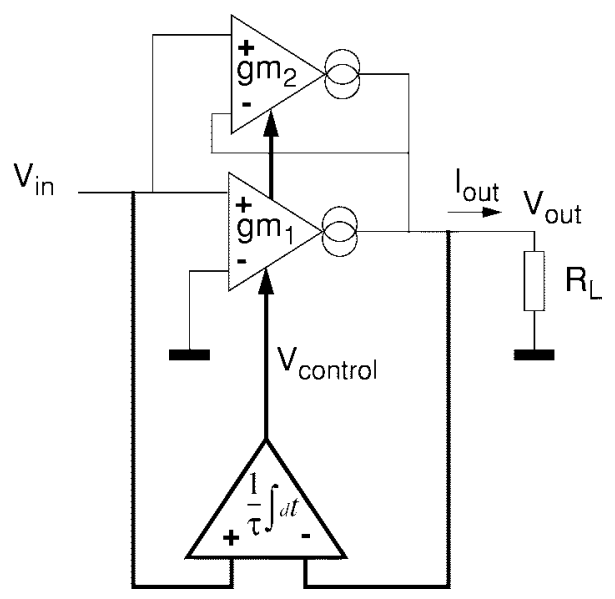

(c)

Fig. 2. The adaptive line driver principle in three steps. (a) A single transconductor $g m_{1}$ has an infinite output impedance. (b) Adding $g m_{2}$ results in an output impedance equal to $1 / \mathrm{gm}$. (c) The control loop tunes $\mathrm{gm}$ equal to $1 / R_{L}$, and the output impedance is equal to $R_{L}$. No voltage or current is lost in the output impedance of the line driver.

equals one, and neither voltage nor current is lost. However, the output impedance of the circuit of Fig. 2(a) is infinite.

In Fig. 2(b), a second transconductor $g m_{2}$ is added. This transconductor is supposed to be equal to $g m_{1}$ and is connected to the same $V_{\text {control }}$. Now the output resistance of the circuit is

$$
R_{\mathrm{out}}=\frac{1}{g m}
$$

which is equal to $R_{L}$ if $g m=1 / R_{L}$. The gain of the circuit is defined as $V_{\text {out }} / V_{\text {in }}$ in the absence of a reflected wave

$$
\text { gain }=\frac{V_{\text {out }}}{V_{\text {in }}}=\frac{2 g m R_{L}}{1+g m R_{L}} .
$$

If $g m=1 / R_{L}$, the gain is one. $V_{\text {out }}$ of the line driver is now equal to

$$
V_{\text {out }}=V_{\text {in }}+V_{\text {reflect }}
$$

where $V_{\text {reflect }}$ is a reflected wave. If $V_{\text {reflect }}=0$, then $V_{\text {out }}=$ $V_{\text {in }}$ and $g m_{2}$ has zero differential input voltage. The output current of $g m_{2}$ is thus zero. If $V_{\text {reflect }}$ is unequal to zero, then $g m_{2}$ only sees the reflected wave and $g m_{2}$ provides the correct output impedance. Therefore, in this configuration, no voltage or current is wasted. ${ }^{1}$ The remaining problem is guaranteeing that $g m$ is equal to $1 / R_{L}$. If $g m=1 / R_{L}$, then $V_{\text {out }}$ equals $V_{\text {in }}$, assuming no reflected waves. This condition is met for $\mathrm{dc}$ and very low frequencies; at high frequencies, reflections may cause $V_{\text {out }}$ to be unequal to $V_{\text {in. }}$. If a control loop is added that controls $\mathrm{gm}$ in such a way that the average values of $V_{\mathrm{in}}$ and $V_{\text {out }}$ are equal, then $g m$ is automatically correct. This is shown in Fig. 2(c). Here, the control loop is implemented with an integrator. The resulting voltage $V_{\text {control }}$ adjusts $\mathrm{gm}$ until it is equal to $1 / R_{L}$. The control loop operates on the $\mathrm{dc}-$ or low frequency - content of the input and output voltages. For this reason, it is important that a dc path exists with correct load resistance from line-driver output to ground. This means the load must be resistive and equal to the characteristic impedance of the line. The load may not contain a coupling capacitor, or transformer. This limits the application of the line driver. For other applications, a different tuning loop, operating at a higher frequency, is needed. Also, for long cables, the dc resistance of the cable will affect the accuracy of the tuning mechanism.

The control loop of Fig. 2(c) has negative feedback as long as the average input voltage is positive. This is easy to guarantee if the buffer is driven by an on-chip signal. If the average input voltage is less than zero, the loop has positive feedback and is unstable. For positive average input signals, the loop can always be made stable as long as the integrator time constant $\tau$ is large enough. Since $\tau$ has no relation to the bandwidth of the line driver, this degree of freedom can be used for making the control loop stable.

Two important properties of a line-driver circuit are the gain and output impedance. Since the line driver of Fig. 2(c) contains a low-frequency control loop, gain and output impedance versus frequency require attention. The gain versus frequency of the circuit of Fig. 2(c) is plotted in Fig. 3(a). The gain is equal to one for all frequencies, assuming that the transconductors have infinite bandwidth. For low frequencies, the gain is one thanks to the control loop, which forces $V_{\text {out }}$ to be equal to $V_{\text {in }}$. At high frequencies, the control loop is inactive and the gain is one according to (2) and $g m=1 / R_{L}$. In conventional line drivers, the gain varies if the output resistance or if the load resistor varies. This new architecture maintains a constant gain.

The output impedance of the line driver can be calculated assuming a reflected wave. The output voltage of the line driver is given by (3), where $V_{\text {in }}$ is held constant. The integrator in Fig. 2(c) generates a control voltage in response to $V_{\text {reflect }}$ equal to

$$
V_{\text {control }}=\frac{-1}{\tau} \int V_{\text {reflect }} d t
$$

\footnotetext{
${ }^{1}$ At the transistor level, the circuit of $g m_{1}$ and $g m_{2}$ needs bias currents, which are lost. How this can be minimized is shown in Section III.
} 
a)

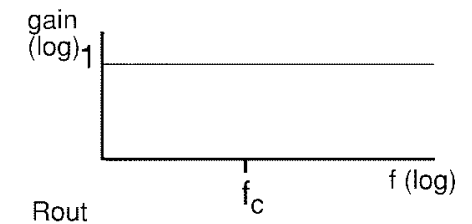

b)

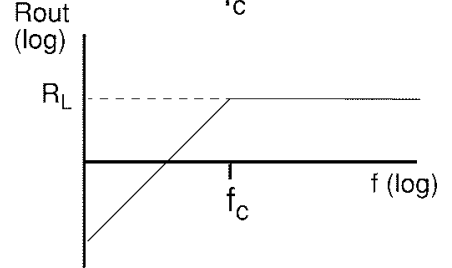

Fig. 3. (a) Voltage gain and output impedance (b) versus frequency of the line driver of Fig. 2(c).

where $\tau$ is the integrator time constant. $V_{\text {reflect }}$ in turn controls $g m_{1}$ and $g m_{2}$ to a value

$$
g m_{1}=g m_{2}=g m_{o}+K_{g m} V_{\text {control }}
$$

where $g m_{O}$ and $K_{g m}$ are constants. The output current $I_{\text {out }}$ [see Fig. 2(c)] of the line driver consists of a constant part ( $I_{\text {outDC }}$ ) and a part depending on $V_{\text {reflect }}$ (or indirectly depending on $V_{\text {reflect }}$ via $\left.V_{\text {control }}\right)$ denoted as $I_{\text {reflect }}$

$$
\begin{aligned}
I_{\text {out }}= & I_{\text {outDC }}+I_{\text {reflect }}=g m_{0} V_{\text {in }}+K_{g m} V_{\text {control }} V_{\text {in }} \\
& -g m_{0} V_{\text {reflect }}-K_{g m} V_{\text {control }} V_{\text {reflect. }} .
\end{aligned}
$$

Since $V_{\text {control }}$ depends on $V_{\text {reflect }}$ as given by (4), it can be seen that

$$
I_{\mathrm{outDC}}=g m_{\circ} \cdot V_{\mathrm{in}} .
$$

Combining (6), (4), and (7), it can be seen that

$$
\begin{aligned}
I_{\text {reflect }}= & \frac{-K_{g m} V_{\text {in }}}{\tau} \int V_{\text {reflect }} d t-g m_{o} \cdot V_{\text {reflect }} \\
& +\frac{K_{g m} V_{\text {reflect }}}{\tau} \int V_{\text {reflect }} d t .
\end{aligned}
$$

Assuming that the first term dominates at low frequencies of $V_{\text {reflect }}$ (i.e., $V_{\text {in }} \gg V_{\text {reflect }}$ ), it can be seen that-with (5)-the output impedance of the line driver of Fig. 2(c) behaves like an inductor $\left(L_{\text {out }}\right)$ with a parallel resistor $\left(R_{L}\right)$

$$
I_{\text {reflect }}=\frac{-1}{L_{\text {out }}} \int V_{\text {reflect }} d t-\frac{V_{\text {reflect }}}{R_{L}}
$$

with

$$
L_{\mathrm{out}}=\frac{\tau}{K_{g m} V_{\mathrm{in}}}
$$

Fig. 3(b) illustrates the output impedance versus frequency. At low frequencies, the output impedance is low due to $L_{\text {out }}$ (the control loop tunes $g m$ in such a way that $V_{\text {out }}$ becomes equal to $V_{\mathrm{in}}$, and thus the output impedance is low). At frequencies higher than a certain corner frequency $f c$, the control loop becomes inactive and the output impedance is equal to $R_{L}$. The corner frequency $f c$ is given by

$$
f_{c}=\frac{R_{L}}{2 \pi L_{\text {out }}}=\frac{K_{g m} R_{L} V_{\mathrm{in}}}{2 \pi \tau} .
$$

At low frequencies, the output impedance is low; however, no reflections will be present (assuming the cable is not

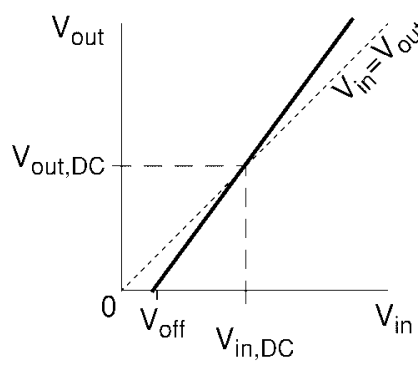

(a)

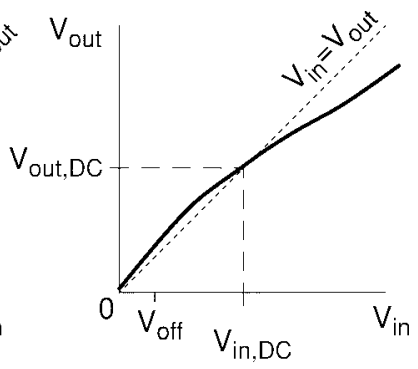

(b)
Fig. 4. (a) Illustration of the effect of offset on differential gain of the circuit of Fig. 2(c). (b) Illustration of the effect of distortion in $g m$ on differential gain.

extremely long). The reflections occurring at high frequencies will be correctly terminated.

The line driver of Fig. 2(c) has the correct output impedance for the frequency range of interest as long as the cable is perfectly terminated. If the cable is perfectly terminated, no reflections occur and the output impedance of the line driver is unimportant. However, if the line is not perfectly terminated (i.e., $Z_{L}$ is unequal to $Z_{\text {cable, where }} Z_{\text {cable }}$ is the cable impedance), a wave is reflected from the receiver side back to the line driver with a reflection coefficient $\rho$

$$
\rho=\frac{Z_{L}-Z_{\text {cable }}}{Z_{L}+Z_{\text {cable }}}
$$

If the line driver operates correctly, its output impedance is equal to $Z_{L}$ and the returning wave is reflected at the linedriver output back to the receiver side with the same reflection coefficient as given by (12). The result is that the receiver side sees a reflected wave of $\rho^{2} V_{\mathrm{in}}$, which is small for reasonable matching of $R_{L}$ to the cable. Therefore, it makes sense to have an adaptive output impedance, even if the cable is not perfectly terminated.

Offset and distortion in the transconductors affect the behavior of the tuning loop. If $g m_{1}$ or $g m_{2}$ have an offset voltage $V_{\text {off }}$, then the control loop will tune the differential gain to a value not equal to one. This is illustrated in Fig. 4(a). If the average input voltage is equal to $V_{\mathrm{inDC}}$, then the loop controls $g m$ in such a way that the average output voltage $V_{\text {out,DC }}$ is

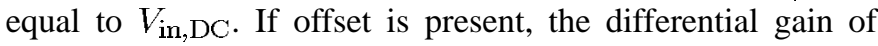
the circuit around $V_{\mathrm{in}, \mathrm{DC}}$ for small-signal frequencies higher than $f_{c}$ is

$$
\text { difgain }=\frac{V_{\mathrm{in}, \mathrm{DC}}}{V_{\mathrm{in}, \mathrm{DC}}-V_{\mathrm{off}}} .
$$

For example a $1-\mathrm{mV}$ offset with $V_{\mathrm{in}, \mathrm{DC}}=100 \mathrm{mV}$ results in a differential gain of 1.01 instead of 1 . The error in differential gain is $1 \%$.

Another nonideality is distortion in the transfer function of $g m_{1}$. This is illustrated in Fig. 4(b). The control loop tunes $g m$ so that $V_{\mathrm{in}, \mathrm{DC}}=V_{\text {out,DC }}$. Since the transfer function for the combination of $g m_{1}$ and $R_{L}$ is nonlinear, the differential gain will be unequal to one for signal frequencies higher than $f_{c}$. Distortion in $g m_{2}$ only affects the nonlinearity of the output impedance. Since $g m_{2}$ sees only a small $V_{\text {reflect, }}$, this is not 


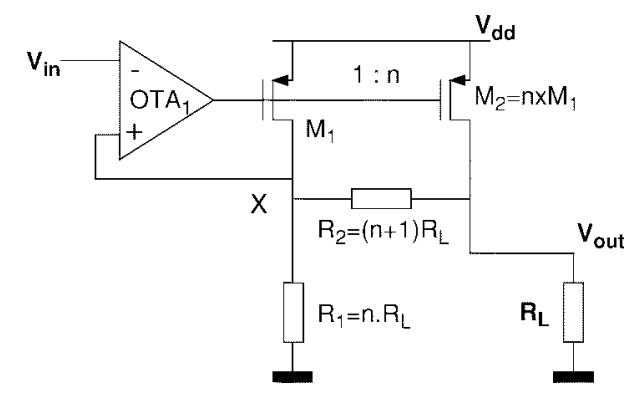

Fig. 5. Video line driver that is not yet tunable.

generally a problem. An analytical expression for predicting the differential gain error due to distortion in $g m_{1}$ is more complex since this effect depends on the type of distortion.

\section{ANAlog VIDEO LINE DRIVER}

In this section, a line driver is described for use as an analog output buffer for a resistive video digital-to-analog converter [1]. The technology is a standard digital $0.35-\mu \mathrm{m}$ CMOS. The analog output voltage range is $0-1.4 \mathrm{~V}$, with a THD less than $-50 \mathrm{~dB}$ for a $1.2-\mathrm{V}_{\mathrm{pp}}$ signal up to $5 \mathrm{MHz}$. The cable impedance is fixed at $75 \Omega$, i.e., the line driver does not have to drive cables with different impedances.

In general, there is a tradeoff between linearity and tuning range for continuously tunable transconductors [5]. A high linearity results in a small tuning range, and a large tuning range has the drawback of poor linearity. To make an optimum circuit for this application, we chose to tune only for process variations. The transconductor must be tunable over such a range that the $g m$ can be tuned to $13.3 \mathrm{~mA} / \mathrm{V}(1 / 75 \mathrm{~A} / \mathrm{V})$ for all process spreads and temperature variations. For the process used, this tuning range is $\pm 30 \%$.

If in Fig. 5 the resistor $R_{2}$ is ignored, this circuit is the implementation of the circuit in Fig. 2(a). The input voltage is copied to node $x$ by OTA 1 and $M_{1}$. The resulting current flows through $R_{1}$ and is linearly proportional to the input voltage. This current is multiplied by a factor $n$ in $M_{2}$ and driven to the load where a linear current-to-voltage conversion is obtained. The circuit is able to drive down to $0 \mathrm{~V}$ as required. The ratio $n$ is typically large (in this case, 40 ) in order to save power and bias currents in the left half of the circuit. Normally, current mirrors have distortion due to finite output impedances and unmatched drain-to-source voltages. Here, both $M_{1}$ and $M_{2}$ have the same $V_{g s}$ and the same $V_{d s}$ so no distortion due to output impedance is present. If $R_{1}$ is equal to $n \cdot R_{L}$, then the transconductance of the circuit $n / R_{1}=1 / R_{L}$ is correct. Tuning for process spreads in $R_{1}$ will be discussed later.

The next step is to implement the correct output impedance. Instead of adding another transconductor, as has been done in Fig. 2(b), the existing circuitry is used, with the addition of $R_{2}$. If $R_{2}$ is chosen to be equal to $(n+1) R_{L}=R_{1}+R_{L}$, then the output impedance is equal to $R_{L}$. The voltage across $R_{2}$ is equal to $V_{\text {reflect }}$, and the current $V_{\text {reflect }} / R_{2}$ flows through $M_{1}$. This current is multiplied by the factor $n$ of $M_{2}$ to the line driver output. The result is an output impedance equal to $R_{L}$. Note that the output impedance is provided by $R_{2}$, while

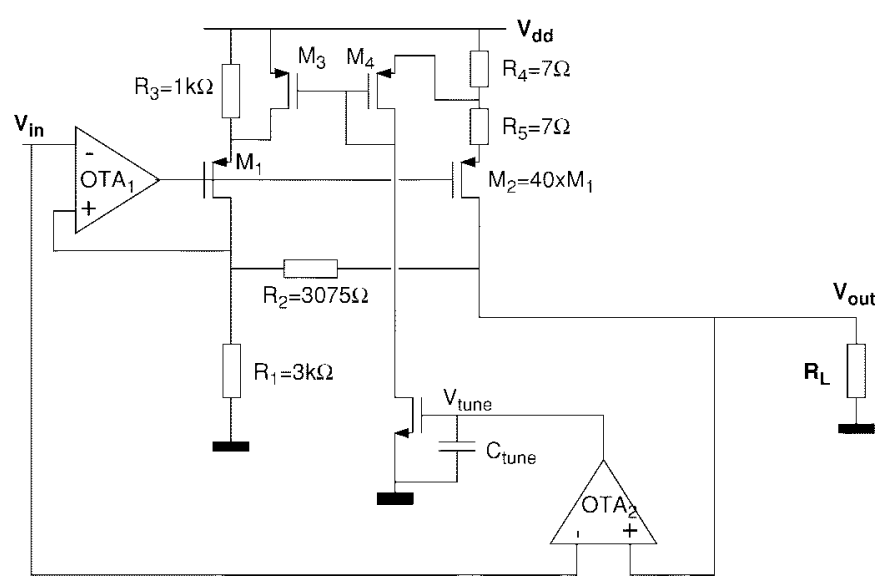

Fig. 6. Adaptive video line driver.

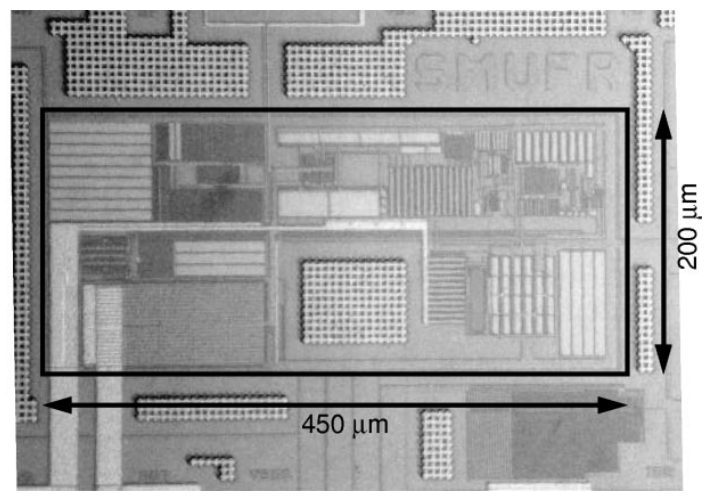

Fig. 7. Chip photograph.

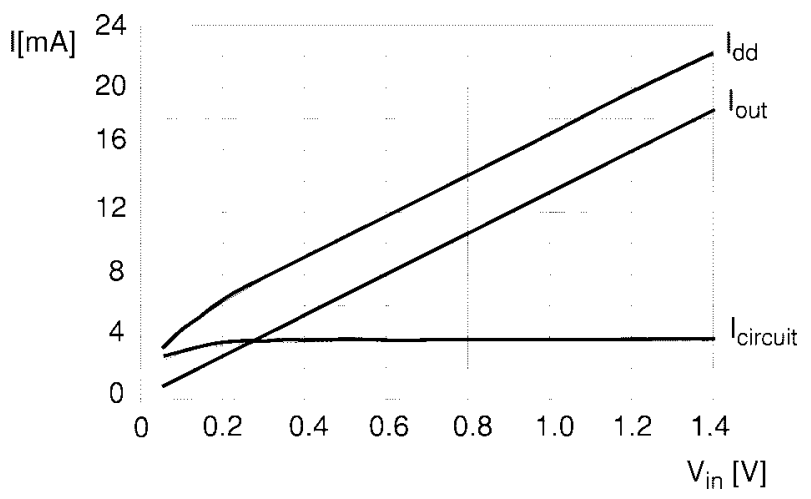

Fig. 8. DC measurements $\left(V_{d d}=2.4 \mathrm{~V}, R_{L}=75 \Omega\right.$ ), various currents versus input voltage.

no signal voltage or current is lost in $R_{2}$ because $R_{2}$ only sees reflections. The circuit of Fig. 5 is based on [6] and [7].

Since the gain and output impedance are still sensitive to process variations in the circuit of Fig. 5, a tuning loop similar to that of Fig. 2(c) must be added. One way to implement a tunable $g m$ is to replace $R_{1}$ and $R_{2}$ with MOSFET's acting as tunable resistors. However, it was difficult to achieve both the tuning range and the linearity, since the voltage over this circuit is relatively large (equal to the full swing input signal, at least for $R_{1}$ ). Therefore, the current ratio in $M_{1}$ and $M_{2}$ is tuned and $R_{1}$ and $R_{2}$ are fixed. An implementation of this principle is shown in Fig. 6 . 


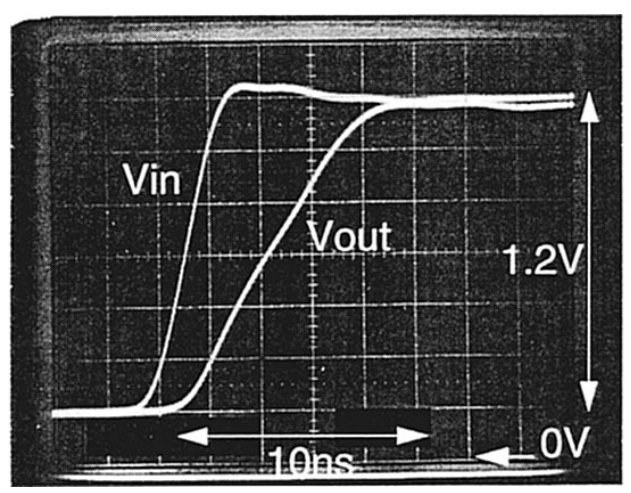

(a)

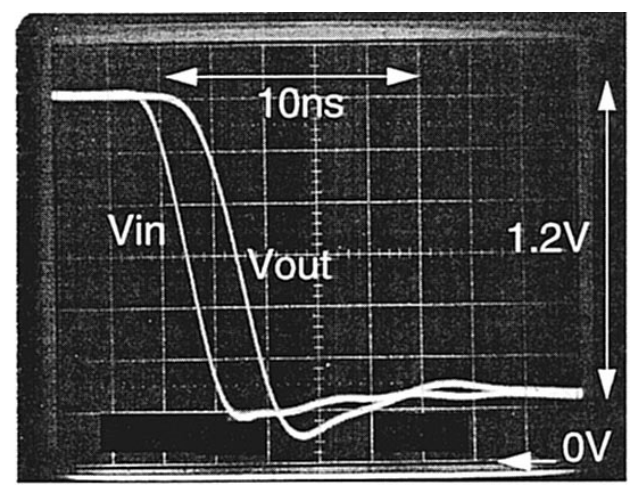

(b)

Fig. 9. Measured step responses $\left(V_{d d}=2.4 \mathrm{~V}, R_{L}=75 \Omega\right.$ ): (a) rising edge and (b) falling edge.

Here, the current ratio is electronically tunable via the voltage, $V_{\text {tune. The }}$ two MOSFET's $\left(M_{1}\right.$ and $\left.M_{2}\right)$ have source degeneration $R_{3}$ in parallel with $M_{3}$ and $R_{4}$ in series with $R_{5}$, respectively. The degeneration resistance of $M_{1}$ is electronically tunable via $V_{\text {tune }} \cdot M_{3}$, operating in the triode range, which behaves like a linear resistor since its gatevoltage variation equals half its drain-voltage variation. The drain current of an MOSFET in triode is given by

$$
I d=\frac{K}{2}\left(2\left(V_{g s}-V_{T}\right) V_{d s}-V_{d s}^{2}\right) .
$$

If $V_{g s}=V_{g s o}+(1 / 2) V_{d s}$, then

$$
I_{d}=K\left(V_{g s o}-V_{T}\right) V_{d s}
$$

and $M_{3}$ acts as a linear, tunable resistor. Due to second-order effects, such as mobility reduction, distortion is present. By shunting $R_{3}$ in parallel to $M_{3}$, a tradeoff in tuning range and linearity is made. Transistor $M_{4}$ is used to generate the $V_{g s 0}$ of $M_{3}$; the resistors $R_{4}$ and $R_{5}$ generate the required $0.5 V_{d s, M 3}$.

The integrator of the control loop is implemented with $\mathrm{OTA}_{2}$, whose output current is integrated in $C_{\text {tune }}$, resulting in $V_{\text {tune }}$; this in turn controls the current ratio. It is important that the bandwidth of the control loop is small. Therefore, $g m_{2} / C_{\text {tune }}$ must be small, where $g m_{2}$ is the transconductance of $\mathrm{OTA}_{2}$. OTA 1 and $\mathrm{OTA}_{2}$ in Fig. 6 are simple folded-cascode amplifiers.

$R_{1}$ and $R_{2}$ are subject to process spreads. However, the control loop tunes the ratio of currents in $M_{1}$ and $M_{2}$ in such a way that the voltage gain is one. Matching between $R_{1}$ and $R_{2}$ will be inherently good thanks to integration. The output impedance of the line driver is slightly lowered by the drain-source resistance $r_{d s 2}$ of $M_{2}$ (operating in saturation). Therefore, $R_{2}$ has been designed to be somewhat larger than $R_{1}+R_{L}$. Deviation from this designed value or spread in $r_{d s 2}$ will result in an error in output impedance and not in a gain error. This error in output impedance, however, is an order of magnitude less than it would be in an integrated nonadaptive line driver.

\section{EXPERIMENTAL RESULTS}

The line driver has been realized in a $0.35-\mu \mathrm{m}$ standard CMOS process. A chip microphotograph is shown in Fig. 7.
The area is $0.09 \mathrm{~mm}^{2}$ including the bias generator. The circuit operates from a 2.4-3.6 V supply voltage. All resistors are implemented in polysilicon. $M_{1}$ is $54 / 0.4$ and $M_{2}$ is $2160 / 0.4$. All measurements described below have been taken at the minimum supply of $2.4 \mathrm{~V}$.

First, the line driver is characterized at dc for a $75-\Omega$ load. This means that the control loop is active and thus $V_{\text {out }}=V_{\text {in }}$. Fig. 8 shows various currents. From this figure, it can be seen that the circuit draws a static current of $3.8 \mathrm{~mA}$, while the output current is up to $19 \mathrm{~mA}$.

Fig. 9 shows the large signal step response of the line driver when driving a $75-\Omega$ load. This slew rate for rising edges is $167 \mathrm{~V} / \mu \mathrm{s}$. The slew rate is limited by $\mathrm{OTA}_{1}$ driving the gate of $M_{2}$. The slew rate is more than sufficient for this application. The falling edge has a larger slew rate since the sink current through $R_{L}$ can be large.

The tuning range of this line driver for large signals has been measured. The tuning range appeared to be from 38 to $85 \Omega$. Note that this tuning range may be different in another processed batch. The tuning range is that which is sufficient to guarantee that $75 \Omega$ is within the tuning range for all process batches. The output voltage range is from 0 to $1.4 \mathrm{~V}$.

The gain versus frequency was measured with an HP 4195 network analyzer. Fig. 10 shows the small signal gain for various loads for $0.7-\mathrm{V}$ dc bias at the input. For low frequencies, the gain is $0 \mathrm{~dB}$ for all loads, thanks to the tuning loop. For frequencies higher than $f_{c}$, which is $4 \mathrm{kHz}$ for $0.7-\mathrm{V}$ input, the gain deviates $0.1 \mathrm{~dB}(1 \%)$ for different loads. This is due to offsets and nonlinearities in the line driver, as has been illustrated in Fig. 4. The small signal bandwidth of the circuit is $130 \mathrm{MHz}$ and is determined by $\mathrm{OTA}_{1}$ with its capacitive loading in Fig. 6.

The output impedance was measured with a HP 4195 network analyzer. Fig. 11 illustrates the output impedance (magnitude and phase) for different loads ranging over the complete tuning range. The dc input voltage is held constant at $0.7 \mathrm{~V}$. For low frequencies, the output impedance is low due to the control loop. For frequencies higher than $f_{c}(4 \mathrm{kHz})$, it is clearly seen that the circuit adapts to the load. The output impedance is resistive and almost equal to the applied load. At high frequencies, the bandwidth of OTA ${ }_{1}$ in combination with its capacitive load results in inductive behavior of the 


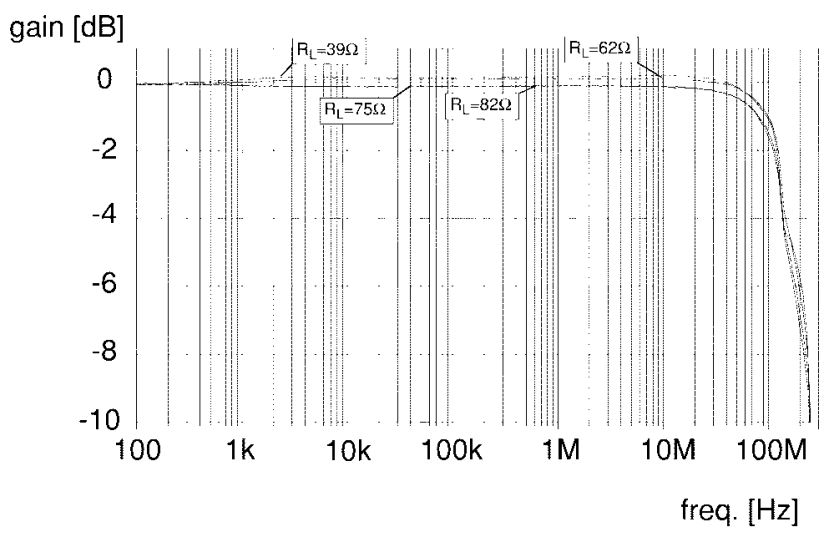

(a)

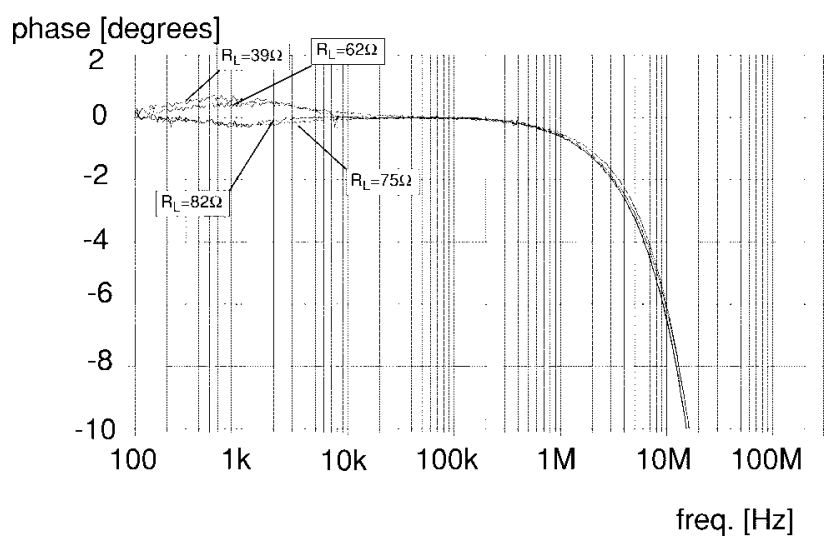

(b)

Fig. 10. (a) Measured voltage gain versus frequency for various loads of the line driver $\left(V_{d d}=2.4 \mathrm{~V}, V_{\text {inDC }}=0.7 \mathrm{~V}\right)$. (b) Measured phase response of the line driver.

TABLE I

Summary OF EXPERIMENTAL Results $\left(V_{d d}=2.4 \mathrm{~V}\right)$

\begin{tabular}{l|l}
\hline parameter & value \\
\hline Vdd range & $2.4 \mathrm{~V} . .3 .6 \mathrm{~V}$ \\
Idd (static) & $3.8 \mathrm{~mA}$ \\
technology & $0.35 \mu \mathrm{m} \mathrm{CMOS}, 5 \mathrm{AL}, 1 \mathrm{PS}$ \\
\hline area & $0.09 \mathrm{~mm}^{2}$ \\
impedance tuning range & $38 . .85 \Omega$ \\
\hline output voltage range & $0 . .1 .4 \mathrm{~V} @ 75 \Omega$ \\
\hline THD $(1.2 \mathrm{Vpp}, 0 . .10 \mathrm{MHz})$ & $<-50 \mathrm{~dB} @ 75 \Omega$ \\
noise $(0 . .5 \mathrm{MHz})$ & $67 \mu \mathrm{V} @ 75 \Omega$ \\
\hline Dynamic range & $77 \mathrm{~dB} @ 75 \Omega$ \\
\hline -3dB bandwidth & $130 \mathrm{MHz}$ \\
Slew rate $($ rise) & $167 \mathrm{~V} / \mu \mathrm{s} @ 75 \Omega$ \\
\hline diff. gain $(\mathrm{f}=4.43 \mathrm{MHz})$ & $2 \% @ 75 \Omega$ \\
\hline diff. phase $(\mathrm{f}=4.43 \mathrm{MHz})$ & $0.5^{\circ} @ 75 \Omega$ \\
\hline
\end{tabular}

output impedance. For the frequency range of interest (up to $10 \mathrm{MHz}$ ), the output impedance is correct.

The distortion was measured with a spectrum analyzer. The distortion versus frequency is plotted in Fig. 12 for $1.2-\mathrm{V}_{\mathrm{pp}}$ sine waves around a $0.7-\mathrm{V}$ dc bias. The distortion is mainly second order and is less than $-50 \mathrm{~dB}$ for frequencies up to $10 \mathrm{MHz}$.

Table I summarizes the experimental results.

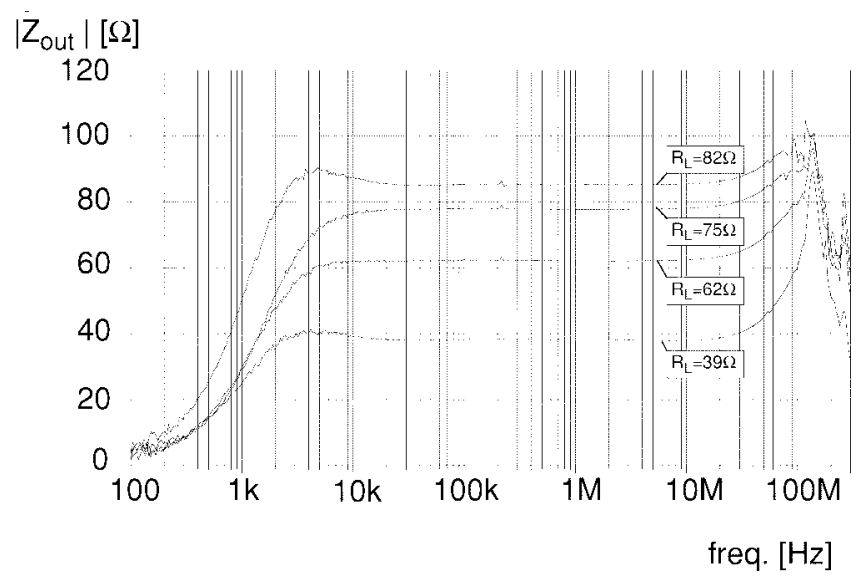

(a)

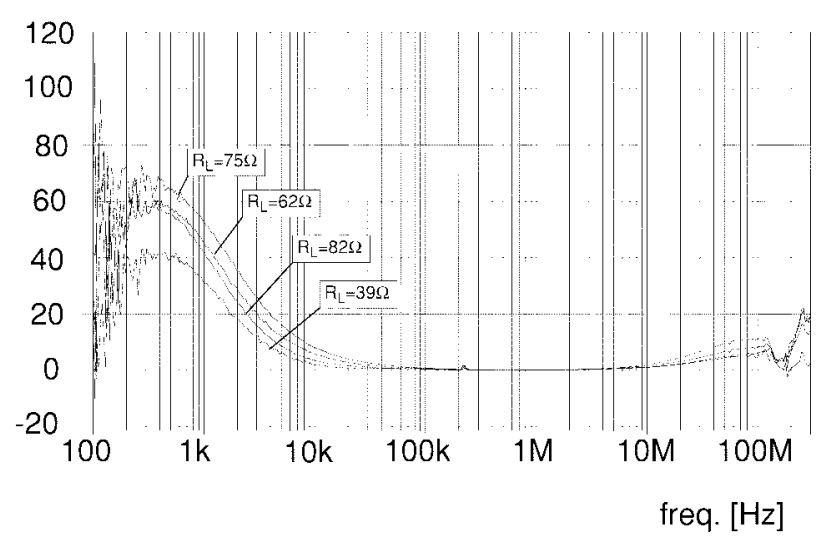

(b)

Fig. 11. (a) Measured magnitude of complex output impedance $Z_{\text {out }}$ as a function of frequency for different loads. ( $\left.V_{d d}=2.4 \mathrm{~V}\right)$. (b) Measured phase of $Z_{\text {out }}$ versus frequency for different loads.

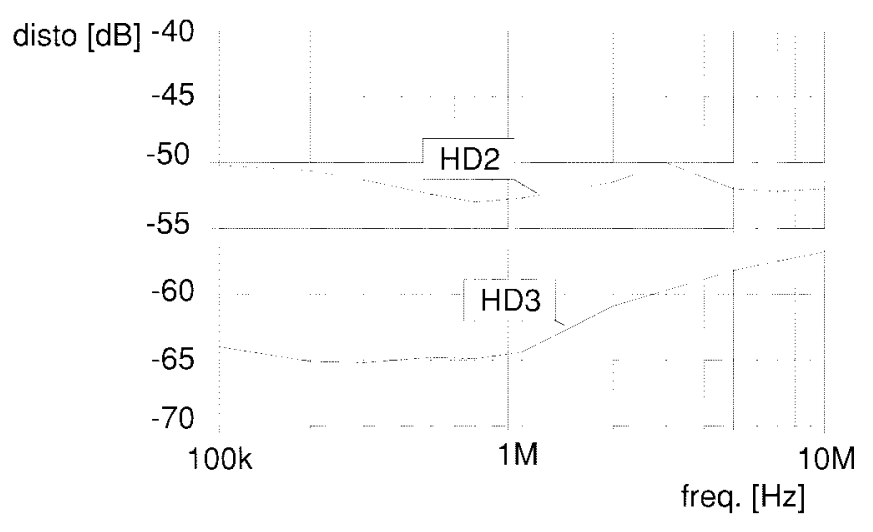

Fig. 12. Measured second and third harmonic distortion versus frequency. $V_{\text {in }}=0.7+0.6 \sin (\omega t)[\mathrm{V}]$

\section{CONCLUSIONS}

A new principle for an adaptive line driver for analog signals has been presented. The main features of this line driver are the absence of voltage or current loss in output impedance and the adaptivity of its output impedance. This adaptive output impedance results in automatically correct output impedances 
for different characteristic impedances. The output impedance is automatically tuned to a value equal to the applied load. So the line-driver output impedance also becomes insensitive to process variations. The effects of some nonidealities have been described.

As an application of the principle, a line driver for analog baseband video signals has been designed in a $0.35-\mu \mathrm{m}$ CMOS technology. The circuit has been designed in such a way that the impedance tuning range is just enough to cover $75 \Omega$ for all process corners. More tuning range would result in more distortion or a more complex circuit. For the given process run, the circuit adapts between 38 - and $85-\Omega$ loads. The circuit operates from a $2.4-\mathrm{V}$ supply voltage and has a THD $<-50$ $\mathrm{dB}$ at $1.2 \mathrm{~V}_{\mathrm{pp}}$ for $0-10 \mathrm{MHz}, 0.09 \mathrm{~mm}^{2}$ area, and $9-\mathrm{mW}$ static power consumption.

\section{ACKNOWLEDGMENT}

The authors would like to thank the reviewers for their comments.

\section{REFERENCES}

[1] M. J. M. Pelgrom, "10-b $50 \mathrm{MHz}$ CMOS D/A converter with 75-ohm buffer," IEEE J. Solid-State Circuits, vol. 25, pp. 1347-1352, Dec. 1990

[2] J. M. Fournier and P. Senn, "A 130-MHz 8-b CMOS video DAC for HDTV applications," IEEE J. Solid-State Circuits, vol. 26, pp. 1073-1077, July 1991.

[3] T. F. Knight and A. Krymm, "A self-terminating low-voltage swing CMOS output driver," IEEE J. Solid-State Circuits, vol. 23, pp. 457-464, Apr. 1988.

[4] T. J. Gabara and S. C. Knauer, "Digitally adjustable resistors in CMOS for high performance applications," IEEE J. Solid-State Circuits, vol. 27, pp. 1176-1185, Aug. 1992.

[5] C. Mensink, B. Nauta, and H. Wallinga, "CMOS soft-switched transconductor and its application in gain control and filters," IEEE J. Solid-State Circuits, vol. 32, pp. 989-998, July 1997.
[6] J. O. Voorman, Active Symmetrical Balance Hybrid Circuit, US patent 4,798,982, Jan. 17, 1989.

[7] S. Baker and E. Swanson, "Amplifier with controlled output impedance," U.S. Patent 5121 080, June 1992.

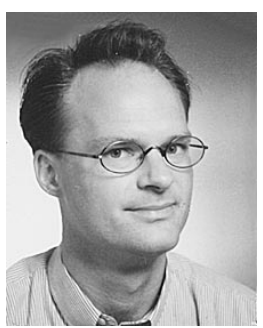

Bram Nauta (''89-M'91) was born in Hengelo, The Netherlands, in 1964. He received the M.Sc. degree (cum laude) in electrical engineering and the Ph.D. degree on the subject of analog CMOS filters for very high frequencies from the University of Twente, Enschede, The Netherlands, in 1987 and 1991, respectively.

In 1991, he joined the Mixed-Signal Circuits and Systems Department of Philips Research, where he worked on high-speed AD converters. From 1994 he led a research group in the same department, working on analog key modules. In 1998, he returned to the University of Twente as a Professor, heading the Department of IC Design in the Department of Electrical Engineering. He also heads the IC Design Group in the MESA research Institute. His Ph.D. dissertation was published as a book: Analog CMOS Filters for Very High Frequencies (Boston, MA: Kluwer, 1993). He holds eight patents in circuit design.

Dr. Nauta received the Shell Study Tour Award for his Ph.D. work. In 1997, he became Associate Editor of IEEE TRANSACTIONS ON CIRCUITS AND Systems Part II: Analog and Digital Signal Processing. In 1998, he was a Guest Editor for the IEEE Journal of Solid-State Circuits.

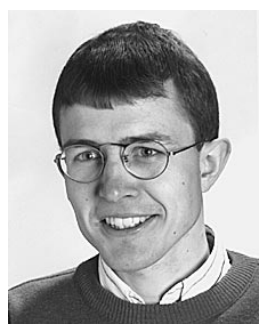

Marcel B. Dijkstra was born in Sint-Maarten (N.H.), The Netherlands, on August 22, 1967. He received the B.S. degree in electrical engineering from the Technical College, Zwolle, The Netherlands, in 1992.

In the same year, he joined Philips Research Laboratories, Eindhoven, The Netherlands, where he has been involved in the design of analog circuits for video applications. In 1997, he joined Philips Optical Storage, Eindhoven, to work on preamplifiers for optical storage systems. 